\title{
Neurilemomas de cavidad oral y cuello
}

\section{Neurilemmomas of the oral cavity and the neck}

\author{
J.A. García de Marcos' , J.J. Ruiz Masera², A. Dean Ferrer ${ }^{3}$, F. Alamillos Granados', \\ F. Zafra Camacho', G. Barrios Sánchez ${ }^{1}$, A. Vidal Jiménez ${ }^{5}$
}

Resumen: Los neurilemomas son tumores neurogénicos benignos, con origen en la vaina neural. De los neurilemomas extracraneales, aproximadamente un 25 a un $45 \%$ ocurren en cabeza y cuello. La edad de afectación predominante es entre la tercera y la cuarta décadas de vida. El neurilemoma es un tumor claramente circunscrito y, generalmente, de pequeño tamaño. Clínicamente aparece como una masa de crecimiento lento y gradual. El examen microscópico es necesario para el diagnóstico. El tratamiento de elección es la escisión quirúrgica completa tumoral y después de ésta es muy rara su recurrencia.

El propósito de este artículo es presentar un estudio retrospectivo, de nueve casos, de neurilemomas de cavidad oral y cuello, tratados en nuestro servicio de Cirugía Oral y Maxilofacial, entre 1997 y 2001 . Hemos valorado una serie de parámetros epidemiológicos (edad, sexo, localización, tamaño, clínica, estudios previos a la cirugía, tiempo trascurrido entre aparición de síntomas y primera consulta, nervio de origen, patrón histológico predominante (Antoni A, Antoni B), y evolución postquirúrgica. Se ha realizado una revisión de la literatura.

Palabras clave: Neurilemoma; Schwannoma; Cavidad oral; Cuello.

\begin{abstract}
Neurilemomas are benign neurogenic tumours, that derive from the neural sheath. Approximately 25 to 45 per cent of the extracraneal neurilemomas occur in the head and neck region. Neurilemomas usually occur between the third and the fourth decades of life. Neurilemoma is a sharply circumscribed, and usually small tumor. Clinically it manifests as a slow and gradually growing mass; microscopic examination is neccesary for diagnosis. Complete tumoral excision remains the treatment of choice and after this, they rarely recur.

The aim of this article is to report a retrospective study, of nine cases, of oral cavity and neck neurilemomas, treated in our service of Oral and Maxillofacial Surgery, between 1997 and 2001. A series of epidemiological parameters (age, sex, location, size, symptoms, preoperative study, time between the symptom appearance and the first consultation, nerve of origin, predominant histologic pattern (Antoni A, Antoni B) and postoperative evolution) are evaluated. The review of the literature is made.
\end{abstract}

Key words: Neurilemoma; Schwannoma; Oral cavity; Neck.

\footnotetext{
1 Médico Residente, Cirugía Oral y Maxilofacial.

2 Médico Adjunto, Cirugía Oral y Maxilofacial.

3 Médico Adjunto, Cirugía Oral y Maxilofacial.

4 Médico Adjunto.Cirugía Oral y Maxilofacial.

5 Médico Residente. Servicio de Anatomía Patológica.

Hospital Universitario «Reina Sofía». Córdoba, España.
}

\section{Correspondencia:}

J.A. García de Marcos.

C/ Antonio Acuña, 10, 5ㅅ.

28009 Madrid, España.

E-mail: pepio2@hotmail.com 


\section{Introducción}

Los neurilemomas son tumores neurogénicos benignos, de crecimiento lento, derivados de células de la cresta neural. ${ }^{1}$ Se desarrollan a partir de la vaina neural de nervios periféricos motores, sensitivos y simpáticos, y nervios craneales, a excepción de los nervios ópticos y olfatorios craneales, que carecen de vaina de células de Schwann. ${ }^{2}$

En 1910, Verocay describió por primera vez un grupo de tumores neurogénicos a los que se refirió como «neurinomas» (Referido en Colreavy). ${ }^{3}$ En 1920, Antoni describió dos patrones histológicos caracterizados por la hipercelularidad (Antoni A) y la apariencia mixoide (Antoni B) (referido en Hegazy y cols). ${ }^{4}$ En 1935, se propuso que estos tumores podrían originarse a partir de elementos de la vaina nerviosa, denominándose «neurilemomas» (referido en Colreavy). ${ }^{3}$ Además de neurilemomas se han utilizado otros términos para referirse a estos, como Schwannomas o neurinomas. ${ }^{5}$

La cabeza y porción lateral del cuello están afectados en la mayoría de los casos, siendo el origen más común de los neurilemomas intracraneales, el octavo par craneal (neurinoma del acústico). ${ }^{1}$

De los neurilemomas extracraneales, aproximadamente entre un 25 y un $45 \%$ ocurren en cabeza y cuello, pero sólo un $1 \%$ tiene origen intraoral.2,5 La localización más común es en la región laterocervical. ${ }^{2}$

La edad de afectación predominante es entre la tercera y la cuarta décadas de la vida, no estando ningún grupo de edad exento, pudiéndose afectar desde niños a ancianos de más de 80 años. ${ }^{2}$

El neurilemoma es un tumor claramente circunscrito y generalmente encapsulado, con forma oval, redondeada o fusiforme. ${ }^{1,2}$

La mayoría de los pacientes refiere la aparición de una masa, de crecimiento gradual, no dolorosa, sin síntomas neurológicos, pudiendo estar presente años antes de que el paciente solicite atención médica. $2,3,6$

El diagnóstico preoperatorio del neurilemoma es raro, ${ }^{2}$ aunque la angiografía intravenosa de sustracción digital, la Tomografía Computerizada (TC) y la Resonancia Magnética (RM) aumentan la probabilidad de realizarlo. ${ }^{1}$

El diagnóstico se confirma siempre con un examen microscópico y el tratamiento de elección es la escisión quirúrgica.1,5

Los neurilemomas muy raramente sufren degeneración maligna y después de la escisión completa es muy rara su recurrencia.2,5

El objetivo del presente trabajo es presentar una serie de casos de neurilemomas de cavidad oral y cuello, así como una revisión de la literatura.

\section{Material y método}

Presentamos un estudio retrospectivo de nueve casos, tratados en nuestro Servicio de Cirugía Oral y Maxilofacial, del Hospital Universitario Reina Sofía de Córdoba, entre noviembre de 1997 y abril del 2001, con diagnóstico de neurilemoma. En todos los casos se valoró: edad, sexo, localización, tamaño, clínica, estudios previos a la cirugía, tiempo transcurrido entre aparición de síntomas y primera consulta, patrón histológico predominante (Antoni A, Anto-

\section{Introduction}

Neurilemmomas are slow growing, benign neurogenic tumors that arise from the neural crest. They develop from the neural sheath of the peripheral motor, sensory and sympathetic nerves and from the cranial nerve pairs, with the exception of the optic and olfactory nerves that do not have a schwann cell sheath. ${ }^{2}$

In 1910 Verocay first described a group of neurogenic tumors that he referred to as «neurinomas» (referred to by Colreavy3). In 1920 Antoni described two histological patterns characterized by being hypercelluar (Antoni A), and with a myxoid appearance (Antoni B) (referred to by Hegazy et al. $\left.{ }^{4}\right)$. In 1935 it was proposed that these tumors could originate from elements of the nerve sheath, and they became known as «Neurilemmomas» (referred to by Colreary3). In addition to neurilemmomas other terms have been used to refer to them, such as schwannomas or neurinomas. ${ }^{5}$

The head and lateral portion of the neck are affected in most cases, and the most common source of intracranial neurilemmomas is cranial nerve VIII (acoustic neurinomas). ${ }^{1}$

Of the extracranial neurilemmomas, approximately 25 to $45 \%$ occur in the head and neck, but only $1 \%$ have an intraoral origin. 2,5 The most common localization is in the laterocervical region. ${ }^{2}$

It is predominant in the third and fourth decades of life, and no age group is exempt. It can affect from children to adults over the age of $80 .{ }^{2}$

The neurilemmoma is a sharply defined and generally encapsulated tumor, with an oval shape, either rounded or fusiform. ${ }^{1,2}$

Most patients report the appearance of a slow-growing, painless mass, with no neurological symptoms, which can exist for years before the patient seeks medical attention. 2,3,6

The preoperative diagnosis of the neurilemmoma is rare, ${ }^{2}$ although with digital intravenous subtraction angiography, Computed Tomography (CT) scans and Nuclear Magnetic Resonance, the probabilities are increased.

The diagnosis is always confirmed with a microscopic examination and the treatment of choice is surgical excision. 1,5

Neurilemmomas very rarely experience malignant degeneration and after complete excision recurrence is very rare., 2,5

The object of the present work is to present a series of cases of neurilemmomas of the oral cavity and neck, as well as a revision of the literature.

\section{Material and method}

We present a retrospective study of nine cases, treated in our Oral and Maxillofacial Service at the University Hospital Reina Sofía, Córdoba between November 1997 and April 2001, with a diagnosis of neurilemmoma. In all 
ni B), nervio de origen y evolución postquirúrgica.

\section{Resultados}

La Tabla 1, muestra las características de los casos estudiados.

De los nueve casos cinco eran mujeres y cuatro hombres. El rango de edad estaba entre 14 y 77 años, siendo la edad media de 29,33 años (Tabla 1).

El tiempo transcurrido entre la aparición de síntomas y la primera consulta varía desde 7 días a 10 años, con una media de 1,94 años.

Las zonas de localización más frecuente fueron la cervical $(33,3 \%)$ y la lingual $(33,3 \%)$, con tres casos cada una, afectándose una sola vez el espacio parafaringeo $(11,11 \%)$, labio superior $(11,11 \%)$ y encía vestibular mandibular $(11,11 \%)$.

En todos los casos la manifestación clínica del tumor, fue la aparición de una masa, existiendo además disfagia en un caso y dolor ocasional en otro caso (Fig. 1).

En cinco casos, todos de localización intraoral, no se utilizó ninguna prueba diagnóstica previa a la cirugía $(55,5 \%)$ y en el resto, todos de localización cervical, se utilizó PAAF y TC en tres casos $(33,3 \%)$, y TC y biopsia en un caso $(11,1 \%)$ (Fig. 2).

En todos los casos se llevó a cabo un tratamiento quirúrgico, que consistió en la extirpación completa del tumor, con la conservación del nervio o los nervios involucrados, cuando estos se objetivaban. El caso número 4 se acompañó de una submaxilectomía. En el caso 5, de localización parafaringea, se realizó una biopsia previa transfaringea por vía oral. Para la resección de este tumor se realizó un abordaje mediante osteotomía mandibular parasinfisaria (Fig. 3).

Sólo fue posible determinar el nervio de origen en los casos de localización submaxilar y yugulodigástrica, donde la tumoración dependía del nervio lingual y del nervio espinal respectivamente.

El tamaño de las lesiones oscilaba entre $2 \mathrm{~mm}$, la más pequeña, y $6 \mathrm{~cm}$, la más grande, con una media de $2,47 \mathrm{~cm}$ (Fig. 4).

El patrón histológico predominante fue el Antoni $A$, existiendo una proporción de éste con respecto al patrón Antoni $B$ de más del $80 \%$ en cinco casos $(55,5 \%)$, entre 60 y $80 \%$ en dos casos $(22,2 \%)$ y entre 50 y $60 \%$ en un caso (11,1\%) (Fig. 5). Sólo en un caso $(11,1 \%)$ existió predominio del patrón Antoni $B$ con respecto al Antoni $A$, siendo el porcentaje superior al $80 \%$ (caso 3 tabla 1) (Fig. 6). cases the following were taken into account: age, sex, localization, size, clinical signs, preoperative evaluations, time between the appearance of the symptoms and the first consultation, predominant histological pattern (Antoni A, Antoni $B)$, nerve of origin and postoperative evolution.

\section{Results}

Table 1 shows the characteristics of the cases studies. Of the nine cases five were women and four were men. The age range was between 14 and 77 , and the average age was 29.33 (Table 1). The time that had passed from the appearance of symptoms and the first consultation varied from 7 days to 10 years, with an average of 1.94 years.

The areas where there was a greater number of localizations was the neck (33.3\%) and the tongue (33.3\%), each with three cases, while the parapharyngeal space was affected only once (11.11\%), upper lip (11.11\%) and gums of the mandibular cavity (11.11\%).

In all cases the clinical manifestation of the tumors was the appearance of a mass, together with dysphagia in one case and occasional pain in another (Fig. 1).

In five cases all the localizations were intraoral and no preoperative diagnostic tests were carried out (55.5\%). The remainder were all cervical localizations, and FNA and CT scans were used in three cases (33.3\%), and CT scan and biopsy were used in one case (11.1\%) (Fig. 2).

In all cases surgical treatment was carried out, which consisted in the complete excision of the tumor, with the conservation of the nerve or nerves involved when these were affected. Case number 4 underwent a sub-maxillectomy. Case 5, with a parapharyngeal localization, underwent a previous oral transpharyngeal biopsy. For the resection of this tumor the approach was made by means of a parasynfysial osteotomy of the mandible (Fig. 3).

Determining the nerve of origin was only possible in those 
Tabla 1.

\begin{tabular}{|c|c|c|c|c|c|c|c|c|c|c|}
\hline Caso & Edad & Sexo & Localización & Tamaño & Clínica & $\begin{array}{l}\text { Estudios } \\
\text { previos } \\
\text { a la cirugía }\end{array}$ & $\mathrm{T}$ & $\begin{array}{c}\text { Nervios de } \\
\text { origen }\end{array}$ & $\begin{array}{c}\text { Patrón } \\
\text { histológico } \\
\text { predominante }\end{array}$ & $\begin{array}{l}\text { Evolución } \\
\text { postcirugía }\end{array}$ \\
\hline 1 & 34 & Hombre & $\begin{array}{l}\text { Laterocervical } \\
\text { posterior }\end{array}$ & $\begin{array}{l}4 \mathrm{~cm} \text { de } \\
\text { diámetro mayor }\end{array}$ & Masa & TC, PAAF & 4 meses & Indeterminado & $\begin{array}{l}50-60 \% \text { de } \\
\text { predominio } \\
\text { de Antoni A. }\end{array}$ & $\begin{array}{c}\text { No } \\
\text { recidiva tras } \\
7 \text { años }\end{array}$ \\
\hline 2 & 14 & Hombre & Lengua & $8 \times 4 \mathrm{~mm}$ & Masa & Ninguno & 2 meses & Indeterminado & $\begin{array}{c}\text { Más del } 80 \% \\
\text { de predominio } \\
\text { de Antoni A. }\end{array}$ & $\begin{array}{l}\text { No } \\
\text { recidiva tras } \\
7 \text { años }\end{array}$ \\
\hline 3 & 31 & Mujer & Yugulodigástrica. & $6 \times 3 \times 3 \mathrm{~cm}$ & Masa & TC, PAAF & 4 años & Nervio espinal & $\begin{array}{c}\text { Más del } 80 \% \\
\text { de predominio } \\
\text { de Antoni B. }\end{array}$ & $\begin{array}{l}\text { No } \\
\text { recidiva tras } \\
4 \text { años }\end{array}$ \\
\hline 4 & 31 & Mujer & Submaxilar & $2,8 \times 1,4 \mathrm{~cm}$ & Masa & TC, PAAF & 6 meses & Nervio lingual & $\begin{array}{l}\text { Más del } 80 \% \\
\text { de predominio } \\
\text { de Antoni A. }\end{array}$ & $\begin{array}{l}\text { No } \\
\text { recidiva tras } \\
4 \text { años }\end{array}$ \\
\hline 5 & 16 & Hombre & $\begin{array}{c}\text { Espacio } \\
\text { parafaringeo }\end{array}$ & $\begin{array}{c}6 \mathrm{~cm} \text { de } \\
\text { diámetro mayor }\end{array}$ & $\begin{array}{l}\text { Masa } \\
\text { Disfagia }\end{array}$ & TC, biopsia & 7 días & Indeterminado & $\begin{array}{l}60-80 \% \text { de } \\
\text { Antoni A }\end{array}$ & $\begin{array}{c}\text { No } \\
\text { recidiva tras } \\
2 \text { años }\end{array}$ \\
\hline 6 & 24 & Mujer & Lengua & $\begin{array}{l}2 \mathrm{~mm} \text { de } \\
\text { diámetro mayor }\end{array}$ & Masa & Ninguno & 10 años & Indeterminado & $\begin{array}{l}60-80 \% \text { de } \\
\text { Antoni A }\end{array}$ & $\begin{array}{c}\text { No } \\
\text { recidiva tras } \\
3 \text { años }\end{array}$ \\
\hline 7 & 14 & Hombre & Lengua & $\begin{array}{c}1 \mathrm{~cm} \text { de } \\
\text { diámetro mayor }\end{array}$ & Masa & Ninguno & 6 meses & Indeterminado & $\begin{array}{c}\text { Más del } 80 \% \\
\text { de predominio } \\
\text { de Antoni A }\end{array}$ & $\begin{array}{c}\text { No } \\
\text { recidiva tras } \\
7 \text { años }\end{array}$ \\
\hline 8 & 23 & Mujer & Labio superior & $\begin{array}{c}1 \mathrm{~cm} \text { de } \\
\text { diámetro mayor }\end{array}$ & $\begin{array}{c}\text { Masa } \\
\text { Dolor } \\
\text { ocasional }\end{array}$ & Ninguno & 1 año & Indeterminado & $\begin{array}{c}\text { Más del } 80 \% \\
\text { de predominio } \\
\text { de Antoni A }\end{array}$ & $\begin{array}{l}\text { No } \\
\text { recidiva tras } \\
4 \text { años }\end{array}$ \\
\hline 9 & 77 & Mujer & $\begin{array}{c}\text { Encía vestibular } \\
\text { mandibular }\end{array}$ & $5 \times 2 \mathrm{~mm}$ & Masa & Ninguno & 1 año & Indeterminado & $\begin{array}{c}\text { Más del } 80 \% \\
\text { de predominio } \\
\text { de Antoni A }\end{array}$ & $\begin{array}{c}\text { No } \\
\text { recidiva tras } \\
3 \text { años }\end{array}$ \\
\hline
\end{tabular}

Table 1.

\begin{tabular}{|c|c|c|c|c|c|c|c|c|c|c|}
\hline Case & Age & Sex & Location & Size & $\begin{array}{l}\text { Clinical } \\
\text { signs }\end{array}$ & $\begin{array}{l}\text { Preop. } \\
\text { studies }\end{array}$ & $\mathrm{T}$ & $\begin{array}{l}\text { Nerve of } \\
\text { origin }\end{array}$ & $\begin{array}{c}\text { Predominant } \\
\text { histologic } \\
\text { pattern }\end{array}$ & $\begin{array}{l}\text { Postop. } \\
\text { evolution }\end{array}$ \\
\hline 1 & 34 & Male & $\begin{array}{c}\text { Posterior } \\
\text { Laterocervical }\end{array}$ & $\begin{array}{l}\text { Largest diameter } \\
\qquad 4 \mathrm{~cm}\end{array}$ & Mass & CT, FNA & 4 months & Undetermined & $\begin{array}{l}50-60 \% \\
\text { predominance } \\
\text { of Antoni A. }\end{array}$ & $\begin{array}{c}\text { No } \\
\text { Recurrence } \\
\text { after } 7 \text { years }\end{array}$ \\
\hline 2 & 14 & Male & Tongue & $8 \times 4 \mathrm{~mm}$ & Mass & None & 2 months & Undetermined & $\begin{array}{c}\text { More than } 80 \% \\
\text { predominance } \\
\text { of Antoni A. }\end{array}$ & $\begin{array}{c}\text { No } \\
\text { Recurrence } \\
\text { after } 7 \text { years }\end{array}$ \\
\hline 3 & 31 & Female & $\begin{array}{l}\text { Jugular- } \\
\text { digastric }\end{array}$ & $6 \times 3 \times 3 \mathrm{~cm}$ & Mass & CT, FNA & 4 years & Spinal nerve & $\begin{array}{c}\text { More than } 80 \% \\
\text { predominance } \\
\text { Antoni B. }\end{array}$ & $\begin{array}{c}\text { No } \\
\text { Recurrence } \\
\text { after } 4 \text { years }\end{array}$ \\
\hline 4 & 31 & Female & Submaxillary & $2,8 \times 1,4 \mathrm{~cm}$ & Mass & FNA, CT & 6 months & Lingual nerve & $\begin{array}{c}\text { More than } 80 \% \\
\text { predominance } \\
\text { of Antoni A. }\end{array}$ & $\begin{array}{c}\text { No } \\
\text { Recurrence } \\
\text { after } 4 \text { years }\end{array}$ \\
\hline 5 & 16 & Male & $\begin{array}{c}\text { Parapharyngea } \\
\text { space }\end{array}$ & $\begin{array}{c}\text { Largest } \\
\text { diameter } 6 \mathrm{~cm}\end{array}$ & $\begin{array}{c}\text { Mass } \\
\text { Dysphagia }\end{array}$ & CT, biopsy & 7 days & Undetermined & $\begin{array}{c}60-80 \% \\
\text { of Antoni A }\end{array}$ & $\begin{array}{c}\text { No } \\
\text { recurrence } \\
\text { after } 2 \text { years }\end{array}$ \\
\hline 6 & 24 & Female & Tongue & $\begin{array}{c}\text { Largest } \\
\text { diameter } 2 \mathrm{~mm}\end{array}$ & Mass & None & 10 years & Undetermined & $\begin{array}{c}60-80 \% \\
\text { of Antoni A }\end{array}$ & $\begin{array}{c}\text { No } \\
\text { recurrence } \\
\text { after 3 years }\end{array}$ \\
\hline 7 & 14 & Male & Tongue & $\begin{array}{c}\text { Largest } \\
\text { diameter } 1 \mathrm{~cm}\end{array}$ & Mass & None & 6 months & Undetermined & $\begin{array}{c}\text { More than } 80 \% \\
\text { predominance } \\
\text { of Antoni A }\end{array}$ & $\begin{array}{c}\text { No } \\
\text { recurrence } \\
\text { after } 7 \text { years }\end{array}$ \\
\hline 8 & 23 & Female & Upper lip & $\begin{array}{c}\text { Largest } \\
\text { diameter } 1 \mathrm{~cm}\end{array}$ & $\begin{array}{c}\text { Mass } \\
\text { Occasional } \\
\text { pain }\end{array}$ & None & 1 year & Undetermined & $\begin{array}{l}\text { More than } 80 \% \\
\text { predominance } \\
\text { of Antoni A }\end{array}$ & $\begin{array}{c}\text { No } \\
\text { recurrence } \\
\text { after } 4 \text { years }\end{array}$ \\
\hline 9 & 77 & Female & $\begin{array}{l}\text { Mandibular } \\
\text { cavity gums }\end{array}$ & $5 \times 2 \mathrm{~mm}$ & Mass & None & 1 year & Undetermined & $\begin{array}{l}\text { More than } 80 \% \\
\text { predominance } \\
\text { of Antoni A }\end{array}$ & $\begin{array}{l}\text { No } \\
\text { recurrence } \\
\text { after } 3 \text { years }\end{array}$ \\
\hline
\end{tabular}


En la evolución postoperatoria no se ha evidenciado recurrencia ni complicación en ninguno de los casos, con periodos de seguimiento que van de dos a siete años, siendo la media de seguimiento de 4,5 años.

\section{Discusión}

Los tumores neurógenos constituyen un porcentaje muy bajo dentro de las lesiones neoplásicas de cabeza y cuello. ${ }^{3}$ Este grupo heterogéneo está compuesto de tumores benignos (neurofibromas, neurilemomas, neuromas, nevus neurógeno y mioblastomas de células granulares) y tumores malignos (sarcomas neurogénicos, schawnnomas malignos, neuroepiteliomas y melanomas). ${ }^{3,7}$

En cuanto a la distribución por sexos, hay autores que no encuentran una diferencia significativa entre sexos, y otros que muestran predilección por un sexo determinado. ${ }^{2,8}$ En nuestra serie, de los nueve casos, cinco eran mujeres y cuatro eran hombres.

Casi toda la anatomía de la cabeza y cuello se ha visto afectada por los neurilemomas, incluidos los tejidos blandos de la cara, especialmente la región preauricular, frente, órbita, cuero cabelludo, labios, maxilar, mandíbula, suelo de boca, lengua, senos paranasales, fosa nasal, glándula parótida, nasofaringe y laringe. ${ }^{2}$

Dentro de la localización intraoral, la lengua y el suelo de la boca son las zonas más comúnmente afectadas. 1,5,7,9 Tres de los cinco casos (60\%) intraorales, que presentamos, eran de localización lingual. El paladar, mucosa bucal, labio, y encía también se afectan en orden decreciente. ${ }^{5}$ Raramente aparece como lesión central de los maxilares, más frecuentemente en la mandíbula, siendo el origen de éste el nervio dentario inferior. $9,10,11$

Daly y Roesler, dividen estos tumores, cuando se localizan en cabeza y cueIlo, en dos grupos: medial y lateral. Los tumores laterales provienen de troncos nerviosos cervicales y de los plexos cervical y braquial; los tumores mediales provienen de los últimos cuatro pares cra-

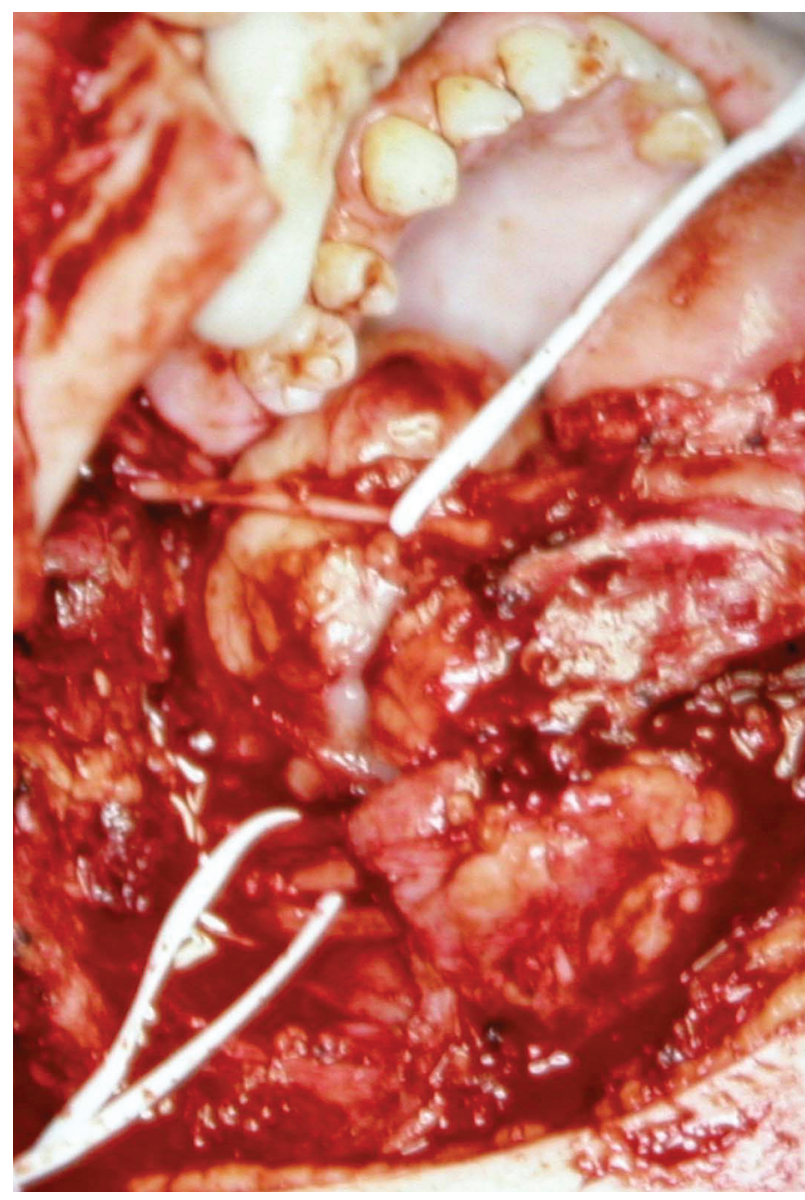

Figura 3. Aspecto intraoperatorio de la lesión tras abordaje de queilotomía media y mandibulotomía parasinfisaria. «Vessel loop» superior separando el nervio lingual (Caso 5).

Figure 3. Intraoperative aspect of the lesion following an approach consisting of a medium cheilotomy and parasymphyseal mandibu lactomy. Upper «vessel loop» separating the lingual nerve (Case 5).

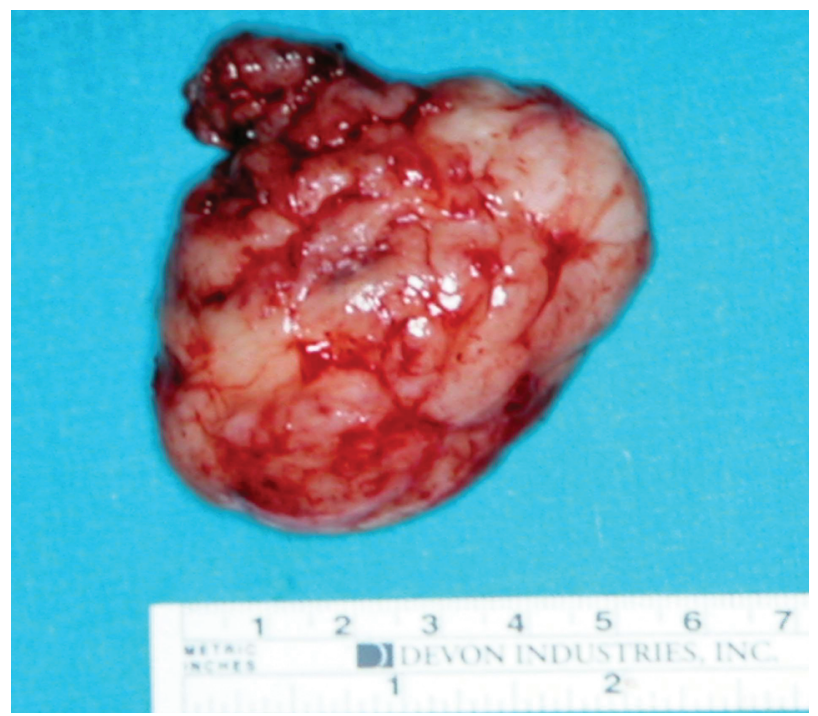

Figura 4. Aspecto de pieza quirúrgica (Caso 5).

Figure 4. Aspect of the surgical specimen (Case 5). cases with submaxillary and jugular digastric localizations, where the tumor depended on the lingual and spinal nerves respectively. The size of the lesions varied between $2 \mathrm{~mm}$, the smallest one, and $6 \mathrm{~cm}$ the largest one. With a average of $2.47 \mathrm{~cm}$ (Fig. 4).

The predominant histological pattern was Antoni A, there being a higher proportion of Antoni $A$ to the Antoni B pattern of more than $80 \%$ in five cases (55.5\%), between 60 and $80 \%$ in two cases (22.2\%), and between 50 and $60 \%$ in one case (11.1\%) (Fig. 5). Only in one case (11.1\%) did the Antoni B predominate the Antoni A pattern, this percentage being more than 80\% (case 3) (Fig. 6). During the postoperative period there was no recurrence or complication in any of the cases, with follow-up periods ranging from two to seven years, the average being 4.5 years.

\section{Discussion}

Neurogenic tumors represent a very low percentage within the neoplasic tumors of the head and neck. ${ }^{3}$ This heterogenic group is composed of benign tumors (neurofibromas, neurilemmomas, neuromas, neurogenic nevus and myoblastomas of granular cells) and malignant tumors (neurogenic sarcomas, malignant schwannomas, neuroepitheliomas and melanomas).3,7

With regard to sex distribution, there are authors that do not find a significant difference between the sexes, and others that show a 
neales y de la cadena simpática cervical, pudiendo manifestarse estos últimos clínicamente como tumores «parafaringeos».1,2 Además, se han clasificado los tumores cervicales, según su relación con el músculo esternocleidomastoideo: del triángulo anterior y del triángulo posterior; y los parafaringeos según su relación con la apofisis estiloides y los músculos que se originan en ésta: del compartimento preestiloideo y del compartimento postestiloideo.8,12

La exacta determinación del nervio originario puede ser imposible de establecer, determinándose en un $50 \%$, el número de casos con correlación con su nervio de origen. ${ }^{2,5}$ En nuestra serie sólo fue posible establecer el nervio de origen, en los casos de localización submaxilar (nervio lingual) y yugulodigástrica (nervio espinal). En el cuello, tanto el vago como los nervios del plexo cervical y braquial son los más frecuentemente afectados cuando el nervio puede reconocerse. $^{2}$

Generalmente es un tumor de consistencia acuosa o fangosa y de pequeño tamaño, entre 0,3 y $3 \mathrm{~cm}$, siendo la mayoría menores de $5 \mathrm{~cm}$, aunque ocasionalmente puede crecer hasta alcanzar los $20 \mathrm{~cm}$ de diámetro. ${ }^{1,2,7}$ En nuestra serie de casos la media del tamaño de las lesiones fue $2,47 \mathrm{~cm}$. Los tumores grandes están irregularmente lobulados y pueden ser parcialmente quísticos, denominándose éstos schawannoma quístico o schwannoma degenerado. ${ }^{1,6} \mathrm{El}$ aumento de tamaño puede estar asociado con una hemorragia intralesional. ${ }^{1}$

Clínicamente, suele aparecer como una masa solitaria, de tamaño variable, no dolorosa, sin síntomas neurológicos, aunque en ocasiones puede comprimir tanto al nervio acompañante como a las estructuras vecinas, produciendo síntomas derivados de la presión de éstas así como dolor y parestesia. 1,2 En nuestra serie el síntoma clínico más frecuente fue la aparición de una masa. Pueden aparecer disfunción de cuerdas vocales, ronquera, tos, dificultad respiratoria y, rara vez, un síndrome de Horner, según la anatomía específica de la región afectada. ${ }^{2}$ Se puede confundir con otras enfermedades de cabeza y cuello, como infecciones o metástasis tumorales. ${ }^{8}$ En el caso número 4, el diagnóstico previo a cirugía, fue tumoración submaxilar, ya que la TC informaba de la existencia de una tumoración en la glándula submaxilar, y la PAAF no fue definitiva.

En nuestro estudio realizamos TC únicamente en los casos cervicales, no siendo necesario como estudio prequirúgico en ninguno de los casos intraorales.

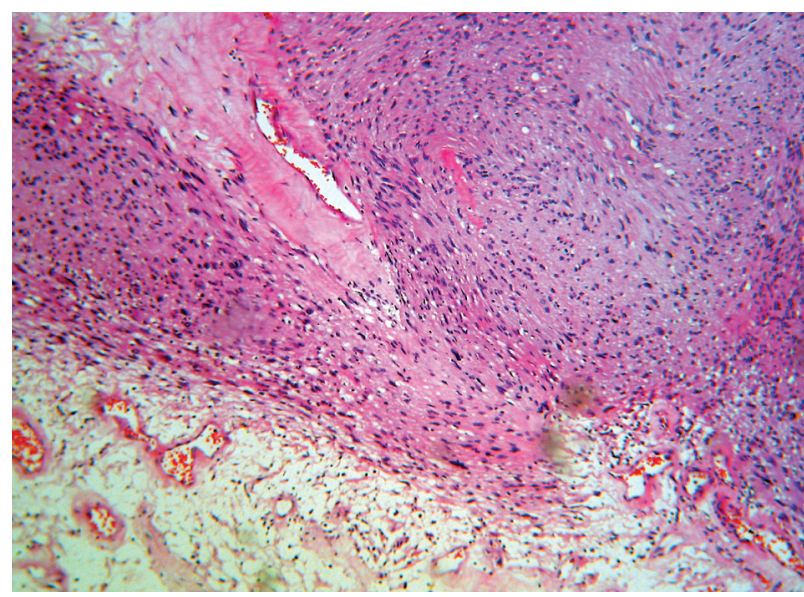

Figura 5. H\&E: arriba: patrón de Antoni A, células fusiformes dispuestas en fascículos; abajo: patrón Antoni B, células separadas vascular.

igure 5. H\&E: top: Antoni A pattern, fusiform cells arranged in fas cicles; bottom: Antoni B pattern, cells separated by the loose matrix, superior right paracentral: vascular hyaline sclerosis.

Figura 6. Distribución del patrón histopatológico Antoni A en nuestra serie de neurilemomas.

Figure 6. Distribution of the histopathological Antoni A pattern in our series of neurilemmomas. predilection for a certain sex. ${ }^{2,8}$ In our series of nine cases, five were women and four were men.

Nearly all the anatomy of the head and neck has been affected by neurilemmomas, including facial soft tissues, especially in the preauricular region, forehead, orbit, scalp, lips, maxilla, mandible, floor of the mouth, tongue, paranasal sinus, nasal fossa, parotid gland, nasopharynx and larynx. ${ }^{2}$

With regard to intraoral localizations, the tongue and the floor of the mouth are areas more commonly affected. 1,5,7,9 Three of the five intraoral cases (60\%) that we present had a lingual localization. The palate, buccal mucosa, lip and gums are also affected in a decreasing order. ${ }^{5}$ Rarely does they appear as a central lesion of the maxillas, and they appear more frequently in the mandible, the origin of this being the inferior dental nerve. $9,10,11$

Daly and Roesler divided these tumors, when located in the head and neck, into two groups: medial and lateral. The lateral tumors emerge from the cervical nerve trunk and from the cervical and branchial plexus; the medial tumors emerge from the last four cranial pair of nerves and from the cervical sympathetic chain, these sometimes appearing clinically as "parapharyngeal» tumors. 1,2 In addition to this, cervical tumors have been classified according to the sternocleidomastoid muscle: from the anterior triangle and from the posterior triangle; and parapharyngeal [tumors] according to the relation with the styloid process and the muscles that originate here: from the prestyloid compartment and the poststyloid8,12 compartment.

Locating the nerve of origin exactly can be impossible, and only about $50 \%$ of cases are correlated with their nerve of origin. ${ }^{2,5}$ In our series we were only able to establish the nerve of origin in submaxillary localizations (lingual nerve) and jugular-digastric (spinal nerve) localizations. In the neck, when the nerve can be identified, it is the vagus as well as the cervical plexus and branchial 
En la TC estas lesiones aparecen como hipodensas en comparación con el músculo, apareciendo cuando se realiza con contraste cierta captación, generalmente periférica (Fig. 7). ${ }^{13}$

La RM puede mostrar no sólo el tumor y su cápsula, sino también, en determinadas casos, el nervio a partir del cual se desarrolla. ${ }^{14}$ Se caracteriza por una señal de igual intensidad a la del músculo en las imágenes ponderadas en T1 y de alta intensidad en T2., 73

El neurilemoma central provoca una imagen radiolúcida, bien delimitada, expansiva y unilocular en la ortopantomografía. 9,10

En la evaluación inicial ha sido recomendado el uso de PAAF; Colreavy y cols. obtienen un diagnóstico preoperatorio definitivo en un 25 por ciento de sus casos. ${ }^{3}$

En nuestro estudio la PAAF fue útil en los casos de localización yugulodigástrica y laterocervical posterior $(66,6 \%$ de los casos en los que se utilizó).

El neurilemoma típico tiene un patrón histológico compuesto de regiones hipercelulares y compactas, áreas de Antoni A, mezcladas con regiones laxas e hipocelulares, áreas de Antoni B.1,2,15

En el patrón Antoni A las células tienen el citoplasma y el núcleo fusiformes, y están dispuestas en fascículos cortos. Las células se pueden disponer alineadas de forma característica formando empalizadas nucleares y cuerpos de Verocay (dos imágenes de empalizadas nucleares enfrentadas entre sí y separadas por una zona acelular). ${ }^{10,15}$

El patrón Antoni B, está formado por células fusiformes similares a las del patrón Antoni $A$, aunque en menor número. En ocasiones la matriz laxa, característica de este patrón, puede degenerar, por cambios mixoides, a un área quística, ${ }^{15}$ como sucedió en el caso numero 3 (Fig. 8).

En nuestra serie existió un claro predominio del patrón Antoni A con respecto al patrón Antoni B, existiendo ambos patrones en todos los casos.

Cuando los cambios degenerativos son pronunciados, se producirán, además de formaciones quísticas, calcificaciones, hialinización, hemorragias y atípias nucleares, denominándose a estos casos neurilemomas antiguos (ancient neurilemomas), no siendo estos cambios determinantes de maliginidad. 2,9

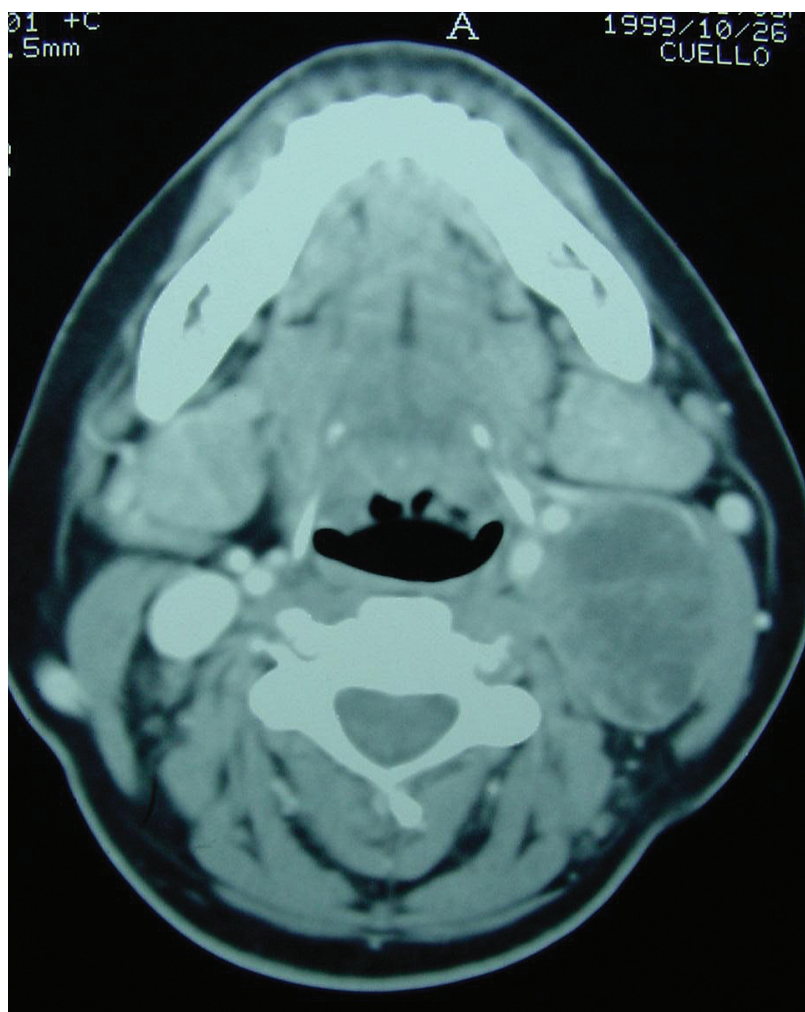

Figura 7. TC del neurilemoma yugulodigástrico, en la que se aprecia la hipodensidad de la lesión con respecto al músculo, así como un aumento de captación periférica (Caso 3).

Figure 7. CT scan of the jugular-digastric neurilemmoma in which the hypodensity of the lesion with respect to the muscle can be appreciated, as well as peripheral enhancement (Case 3).

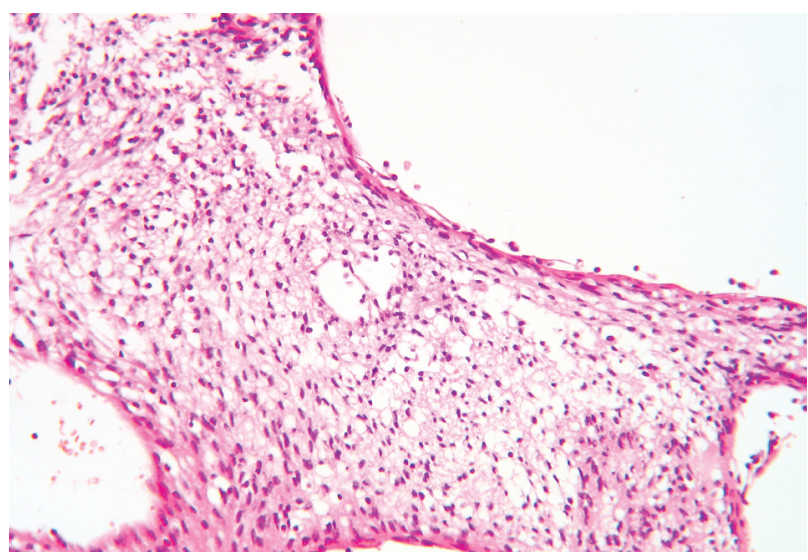

Figura 8. H\&E: patrón Antoni B con degeneración quística en neurilemoma (Caso 3).

Figure 8. H\&E: Antoni B pattern with cystic degeneration in the neurilemmoma (Case 3). nerves that are the most affected. $^{2}$

Generally this tumor has a watery or putty-like consistency. It is small, between 0.3 and $3 \mathrm{~cm}$, most being less than $5 \mathrm{~cm}$, although occasionally it can grow until it reaches a diameter of 20 $\mathrm{cm}{ }^{1,2,7}$ In our case series, the average size of the lesions was of $2.47 \mathrm{~cm}$. The larger tumors are irregularly lobulated and they can be partially cystic, these being called cystic schwannomas or schwannomas with degenerative changes. 1,6 An increase in size can be related to an interlesional hemorrhage. ${ }^{1}$

It appears clinically as a painless, solitary mass, variable in size, without neurological symptoms, although on occasions it can compress the accompanying nerve as well as the neighboring structures, producing symptoms derived from the pressure such as pain and paresthesia. 1,2 In our series the most frequent clinical sign was the appearance of a mass. Dysfunction of the vocal chords, snoring, coughing, breathing difficulties, and rarely Horner's syndrome, may appear depending on the specific anatomy of the affected region. ${ }^{2}$ It can be confused with other complaints of the head and neck, such as infections or metastasic tumors. ${ }^{8}$ In case number 4 the diagnosis previous to surgery, was of a submaxillary tumor, as the CT scan report was of a submaxillary gland tumor and the FNA was not definitive.

In our study we carried out a CT scan only in cervical cases, as a preoperative study was not necessary in any of the intraoral cases.

In the CT scan these lesions appear hypodense compared with the muscle, and when contrast material in introduced 
La microscopía electrónica y el análisis inmunohistoquímico (S100, Leu-7) son útiles para diagnosticar y clasificar de forma precisa los tumores de vainas nerviosas. ${ }^{3}$ Los neurilemomas se marcan intensamente con anticuerpos de la proteína S-100, lo que sirve para diferenciarlo de los tumores de músculo liso, que no se marcan. ${ }^{2}$

Clínica e histológicamente el schwanoma debe ser diferenciado del neurofibroma, que es un tumor neurogénico, procedente también de las células que constituyen la vaina nerviosa. El neurofibroma es más común, está constitiuido por una mezcla de células de schwann y fibroblastos, y tiene mayor tendencia a la malignización. ${ }^{1}$ El neurofibroma es un tumor generalmente no encapsulado, lobulado, de superficie irregular $y$, a diferencia del neurilemoma, que desplaza al nervio asociado, éste se entremezcla con el nervio de origen. $1,2,6,9,16$ Se trata de un tumor de difícil escisión, que recurre o persiste debido a una resección incompleta y en casos de neurofibromatosis hereditaria, puede transformarse en tumor maligno. ${ }^{9}$

El tratamiento de elección es la escisión quirúrgica. ${ }^{1}$ Los neurilemomas que aparezcan en troncos nerviosos mayores, deben ser disecados sin dañar el nervio, ya que las fibras nerviosas no forman parte del tumor, que surge de la envoltura del nervio y desplaza a los axones hacia un lado..$^{2,6}$ Los subtipos no encapsulados requieren un margen de seguridad en la extirpación. ${ }^{1}$ En nuestra serie el tratamiento, en todos los casos, fue la escisión quirúrgica del tumor, conservando el nervio de origen en los dos casos en los que se pudo determinar. No se apreció recidiva del proceso en ninguno de los casos.

Tras la extirpación del neurilemoma pueden producirse secuelas neurológicas transitorias o permanentes, por lo que se debe informar, previa cirugía, sobre esta posibilidad. ${ }^{6,13}$ Leu y cols. consideran prioritaria la conservación y reparación del nervio durante la escisión tumoral, debido a la rara probabilidad de transformación maligna. ${ }^{6}$ La anastómosis término-terminal o la interposición de un injerto nervioso son posibles soluciones cuando sea imposible la preservación del nervio originario. ${ }^{3}$ Se ha sugerido también el uso de técnicas microquirúrgicas para la preservación del nervio y la escisión completa del tumor. ${ }^{14}$

En la revisión de casos de neurilemomas de médula espinal, realizada por Seppälä y cols., a 20 pacientes se les realizó una escisión parcial del tumor, para evitar dañar el nervio de origen. De éstos, 11 sufrieron recurrencia siendo necesaria la reintervención únicamente en dos de ellos. Concluye el estudio que la escisión radical del tumor no está justificada cuando se comprometa la función nerviosa. ${ }^{17}$

\section{Conclusiones}

Los neurilemomas son tumores benignos, derivados de la vaina neural, que se presentan generalmente como una masa de crecimiento lento, que precisan de un examen microscópico para su diagnóstico y cuyo tratamiento es la resección quirúrgica.

\section{Agradecimientos}

Al Dr. Perez-Seoane Orduña (Médico Adjunto. Servicio de Anatomía Patológica. Hospital Universitario Reina Sofía. Córdoba). there is a certain amount of enhancement, generally peripheral'13 (Fig. 7).

NMR can show not only the tumor and the capsule but also, in certain cases, the nerve from which it has developed. ${ }^{14}$ It is characterized by a signal of equal intensity to that of the muscle in $T 1$ weighted sequences and by high signal intensity on $T 2.7,13$

The central neurilemmoma produces a radiolucid, welldefined, expansive and unilocular image in the orthopantomography. 9,10

In the initial evaluation the use of FNA has been recommended. Colreavy and al. obtained a definitive preoperative diagnosis in $25 \%$ of cases. ${ }^{3}$

In our study, FNA was useful in jugular-digastric and posterior laterocervical locations (being used in $66.6 \%$ of cases).

The typical neurilemmoma has a histological pattern composed of dense, hypercellular areas and, Antoni $A$ areas, mixed with loose hypocellular Antoni $B$ areas. $1,2,15$

In the Antoni A pattern, the cytoplasm and nuclei of the cells are fusiform and they are distributed in short fascicles. The cells can be aligned in a characteristic fashion forming nuclear palisades and Verocay bodies (two images of confronted nuclear palisades, separated by an acellular zone). 10,15

The Antoni B pattern is formed of fusiform cells similar to those of the Antoni A pattern, although fewer in number. On occasions the loose matrix, which is a characteristic of this pattern, can degenerate because of myxoid changes, into a cystic area, 15 as occurred in case number 3 (Fig. 8).

In our series there was a clear predominance of the Antoni A pattern over the Antoni $B$, with both patterns existing in all cases.

When degenerative changes are very pronounced, calcifications, hyalinizations, hemorrhages and atypical nuclei will appear as well as cystic formations, these being called ancient neurilemmomas, but these changes do not lead to malignancy. ${ }^{2,9}$

The electronic microscope and the immunohistochemical analysis (S-100, Leu-7) are useful for diagnosing and classifying in a precise way nerve sheath tumors. ${ }^{3}$ Neurilemmomas are stained more strongly with antibodies against the S-100 protein, which serves to differentiate them from smooth muscle tumors, which do not stain. ${ }^{2}$

Clinically and histologically the schwannoma tumor should be differentiated from the neurofribroma, which is a neurogenic tumor that arises from the cells constituting the nerve sheath. The neurofibroma is the most common tumor. It is made up of a mixture of Schwann cells and fibroblasts, and it has a greater tendency to malignancy. The neurofibroma is a tumor which is generally non-encapsulated and lobulated, with an irregular surface and, unlike the neurilemmoma, which pushes away the associated nerve, it becomes intertwined with the nerve of origin $1,2,6,9,16$. It is a tumor which is difficult to remove, that recurs or persists when resection has been incomplete; and in cases of hered- 


\section{Bibliografía}

1. Zachariades N, Skoura C, Papageorgiou G. Giant ancient neurilemmoma of the cervical region: Report of Case. J Oral Maxillofac Surg 2001;59:668-72.

2. Thawley SE, Panje WR, Batsakis G, Lindberg RD. Tumors of the neck. En: Comprehensive management of head and neck tumors. Vol II. Second edition. Philadelphia. W.B.Saunders Company; 1999. pp.1338-40.

3. Colreavy MP, Lacy PD, Hughes J. Head and neck schwanommas-a 10 year review. J Laryngol Otol 2000;114:119-24.

4. Hegazy HM, Snyderman CH, Fan C-Y, Kassam AB. Neurilemmomas of the Paranasal sinuses. Am J Otolaryngol 2001;22:215-18.

5. Pfeifle R, Baur DA, Paulino A. Schwannoma of the tongue: Report of 2 cases. I Oral Maxillofac Surg 2001;59:802-4.

6. Miyawaki T, Nakamura A, Hayashi H. Macrocystic Schwannoma in the seventh cervical nerve. Plast Reconstr Surg 1999;104: 789-92.

7. Flickinger FW, Lozano RL, Yuh WTC, Sachs MA. Neurilemoma of the tongue: MR Findings. J Comput Assist Tomogr 1989;13:886-8.

8. Leu YS, Chang KC. Extracranial Head and Neck Schwannomas: A review of 8 years experience. Acta Otolaryngol 2002;122:435-7.

9. Max RE, Stern D. Benign soft tissue tumors of mesenchymal origin. En: Oral and Maxillofacial Pathology. A rationale for diagnosis and treatment. $1^{\mathrm{a}}$ ed. Illinois: Quintessence publishing, 2003; pp. 395-61.

10. Sapp JP, Eversole LR, Wysocki GP. Lesiones del tejido conjuntivo. En: Patología Oral y Maxilofacial contemporánea. $1^{\underline{a}}$ ed. Madrid : Harcourt Brace, 1998; p.277318.

11. Aguirre JM. Tumores de los maxilares. En: Bagán Sebastián JV, Ceballos Salobreña A, Bermejo Fenoll A, Aguirre Urízar JM, Peñarrocha Diago M. Medicina Oral. 1a ed. Barcelona: Masson, 1995; pp. 503-14.

12. Smoker WRK, Harnsberger HR, Reede DL. Cuello. En: Som PM, Bergeron RT. Radiología de Cabeza y Cuello. Madrid. Ed: Mosby; 1993. p 517.

13. de Vicente Rodriguez JC, Junquera Gutierrez LM, Fresno Forcelledo MF. Neck schwannomas. Med Oral 2003;8(1):71-6.

14. Ku H.-c., Y. C.-w. Review article. Cervical schwannoma: a case report and eight years review. J Laryngol Otol 2000;114:414-7.

15. Lawrence M. Weiss Soft Tissues. En: Weidner, Cote, Sustes, Weiss. Modern Surgical Pathology. Philadel phia. Edit. Saunders; 2003. pp.1834.

16. Muñoz Guerra MF, Rodríguez Campo F, Ortiz de Artiñano F, Sastre Pérez J. Schwannoma of the brachial plexus: an unusual cause for a cervical mass. J Oral Maxillofac Surg 2001; 59:942-4.

17. Seppälä MT, Haltia MJJ, Sankila RJ. Long-term outcome after removal of spinal schwannoma: a clinicopathological study of 187 cases. J Neurosurg 1995;83:6216. itary neurofibromatosis, it can transform into a malignant tumor. ${ }^{9}$

The treatment of choice is surgical removal. ${ }^{1}$ The neurilemmomas that appear in larger nerve trunks, should be dissected without damage to the nerve, as the nerve fibers do not form part of the tumor, that emerges from the capsule of the nerve, pushing the axons aside. ${ }^{2,6}$ The non-encapsulated subtypes require a security margin when removed. ${ }^{1}$ The treatment in our series, in all cases, was surgical excision of the tumor, conserving the nerve of origin in the two cases in which it could be established. In none of the cases did the process recur.

After removal of the neurilemmoma there may be a transitory or permanent neurological sequelae may appear, and information should be given as to this possibility. 6,13 Leu et al. consider the conservation and repair of the nerve a priority during tumor excision, due to the unlikely probability of malignant transformation. ${ }^{8}$ End-to-end anastomosis or nerve graft insertion are possible solutions, when preserving the nerve of origin is impossible. ${ }^{3}$ The use of microsurgical techniques has also been suggested for preserving the nerve and for completely removing the tumor. ${ }^{14}$

In the revision of neurilemmoma cases of the spinal cord, carried out by Seppälä et al., on 20 patients, partial excision of the tumor was performed so as to avoid harming the nerve of origin. Of these, eleven experienced recurrence with reintervention being necessary only in two of them. The study concludes that radical excision of the tumor is not justified if nerve function is compromised. ${ }^{17}$

\section{Conclusions}

Neurilemmomas are benign tumors, derived from the neural sheath, which are generally presented as slow-growing masses. Microscopic examination is required for their diagnosis and treatment entails surgical resection.

\section{Acknowledgements}

To Dr. Pérez-Seoane Orduña (Staff Departament of Pathology. University Hospital «Reina Sofía», Córdoba. 